\title{
A memory-efficient algorithm to obtain splicing graphs and de novo expression estimates from de Bruijn graphs of RNA-Seq data
}

Sing-Hoi Sze $e^{1,2^{*}}$, Aaron M Tarone ${ }^{3}$

From Third IEEE International Conference on Computational Advances in Bio and Medical Sciences (ICCABS 2013)

New Orleans, LA, USA. 12-14 June 2013

\begin{abstract}
Background: The recent advance of high-throughput sequencing makes it feasible to study entire transcriptomes through the application of de novo sequence assembly algorithms. While a popular strategy is to first construct an intermediate de Bruijn graph structure to represent the transcriptome, an additional step is needed to construct predicted transcripts from the graph.

Results: Since the de Bruijn graph contains all branching possibilities, we develop a memory-efficient algorithm to recover alternative splicing information and library-specific expression information directly from the graph without prior genomic knowledge. We implement the algorithm as a postprocessing module of the Velvet assembler. We validate our algorithm by simulating the transcriptome assembly of Drosophila using its known genome, and by performing Drosophila transcriptome assembly using publicly available RNA-Seq libraries. Under a range of conditions, our algorithm recovers sequences and alternative splicing junctions with higher specificity than Oases or Trans-ABySS.

Conclusions: Since our postprocessing algorithm does not consume as much memory as Velvet and is less memory-intensive than Oases, it allows biologists to assemble large libraries with limited computational resources. Our algorithm has been applied to perform transcriptome assembly of the non-model blow fly Lucilia sericata that was reported in a previous article, which shows that the assembly is of high quality and it facilitates comparison of the Lucilia sericata transcriptome to Drosophila and two mosquitoes, prediction and experimental validation of alternative splicing, investigation of differential expression among various developmental stages, and identification of transposable elements.
\end{abstract}

\section{Background}

With the advance of high-throughput sequencing techniques, it is feasible to study entire transcriptomes through the application of de novo sequence assembly algorithms [1-8]. A popular strategy of transcriptome assembly algorithms is to first obtain a de Bruijn graph that contains all branching possibilities [7-10]. An additional step is then performed to construct predicted transcripts from the graph. This strategy is employed by Oases [10] and

\footnotetext{
* Correspondence: shsze@cse.tamu.edu

'Department of Computer Science and Engineering, Texas A\&M University, College Station, TX 77843, USA

Full list of author information is available at the end of the article
}

Trans-ABySS [9], which use output from Velvet [5] and ABySS [6] respectively to obtain predicted transcripts. One drawback of the approach is that Oases can be more memory-intensive than Velvet, which limits its application when computational resources are limited. Alternatively, Trinity [8] uses a different approach of first clustering the data, then constructing an individual de Bruijn graph for each cluster that has simple structure.

We observe that it is possible to develop a memoryefficient algorithm to recover alternative splicing information directly from the intermediate de Bruijn graph structure that contains all branching possibilities (see Figure 1). Although many of the simpler components of

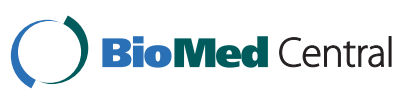

(c) 2014 Sze and Tarone; licensee BioMed Central Ltd. This is an Open Access article distributed under the terms of the Creative Commons Attribution License (http://creativecommons.org/licenses/by/4.0), which permits unrestricted use, distribution, and reproduction in any medium, provided the original work is properly cited. The Creative Commons Public Domain Dedication waiver (http://creativecommons.org/publicdomain/zero/1.0/) applies to the data made available in this article, unless otherwise stated. 


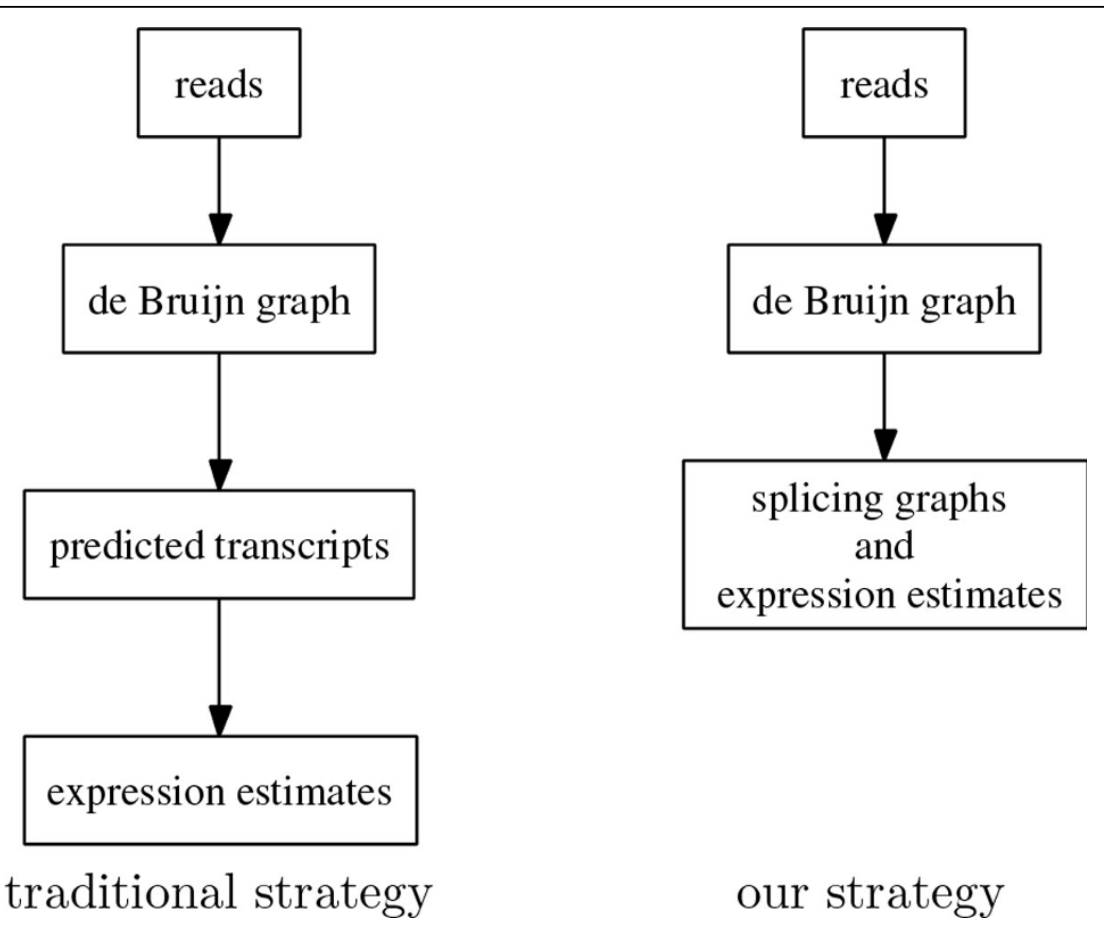

Figure 1 Difference between traditional strategy and our strategy.

the de Bruijn graph can already represent alternatively spliced variants of individual genes, the graph still contains big tangles that need to be addressed. We develop an algorithm to remove the complicated cycles in the de Bruijn graph, and extract acyclic components so that each of them represents a gene and its isoforms in almost all cases. Our goal is to preserve the alternative splicing information that is inherent within the reads as much as possible, and report these components as splicing graphs.

We implement the algorithm as a postprocessing module of Velvet. We validate our algorithm by simulating the transcriptome assembly of Drosophila using its known complete genome under the condition that all gene transcripts have high expression levels, and by performing Drosophila transcriptome assembly using publicly available RNA-Seq libraries. We further employ a de novo expression estimate to simultaneously evaluate the differential expression levels across libraries without requiring any prior knowledge of the genome, which was validated in [11]. We have applied our algorithm to perform transcriptome assembly of the non-model blow fly Lucilia sericata in [11].

\section{Methods}

\section{De Bruijn graph}

Given a set of reads and a parameter $k$, a de Bruijn graph is defined by constructing a vertex for each $k$-mer that appears within the reads. A pair of $k$-mers are connected by a directed edge if the $(k-1)$-suffix of the first $k$-mer is the same as the $(k-1)$-prefix of the second $k$-mer. It has been observed that the de Bruijn graph can be used to implicitly assemble these reads through linking together the same $k$-mer that appears in different reads $[12,13]$. Since the number of vertices and edges in a de Bruijn graph depends on the number of distinct $k$-mers from the reads rather than the total number of reads, this strategy is very popular among short read assembly algorithms for high-throughput sequencing data [2,3,5-7].

\section{Postprocessing algorithm}

In order to retain alternative splicing information, Heber et al [14] developed an EST assembly algorithm that retains all the junctions in the de Bruijn graph. By imposing a $k$-mer coverage cutoff, each component becomes a splicing graph that specifies the alternatively spliced variants of a gene. While this strategy was proved to be successful for EST assembly, there are significant challenges in transcriptome assembly from high-throughput sequencing data that are caused by the shorter reads.

We develop a postprocessing algorithm that extracts the de Bruijn graph from Velvet [5] to construct nonlinear splicing graphs that represent the transcriptome. In order to retain as much alternative path information as possible, Velvet is applied without using the tour bus algorithm that removes the bubbles in the graph, while 
still allowing the removal of short tips. Each node returned from Velvet corresponds to a maximal succession of vertices with no branches.

\section{SNPs}

In order to remove SNPs that are not related to alternative splicing but will create branches in the graph, we search for the following structure: starting from a node, consider the nodes at the end of all its outgoing edges. If the sequences associated with all these nodes are of the same length with long enough matches and very few mismatches, each of these nodes has exactly one outgoing edge that all go to the same final node, there are no other branches going into or out of any of these nodes and no other branches going into the final node, and the structure does not contain a forward node and the corresponding backward node at the same time, we think of all the mismatches within this split-then-merge structure as SNPs. We repeat the procedure at the final node to look for successive split-then-merge structures, and merge all the nodes involved into a single node (see Figure 2 for an example). Note that this strategy only merges together obvious SNPs, and it does not resolve short indels.

\section{Strongly connected components}

We observe that connected regions within the de Bruijn graph that are relatively free of cycles are likely to belong to the same gene. We decompose each connected component into a collection of edge-disjoint strongly connected components, with each strongly connected component being either just a single edge or a maximal subgraph with each vertex reachable from any other vertex. The regions within a strongly connected component that is not just a single edge represent the complicated regions that must contain a cycle, while the remaining regions represent the simpler regions that contain no cycles (see Figure 3 for an example). This

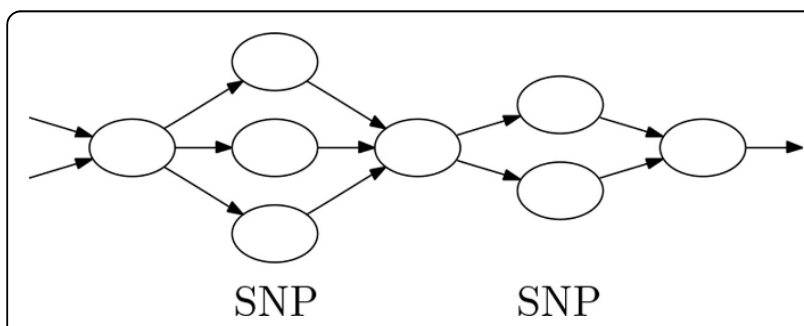

Figure 2 Illustration of two successive sets of nodes that contain SNPs. The sequences within all the nodes in the second column and the sequences within all the nodes on the fourth column must be of the same length in order to contain SNPs. Note that there can be more than one SNP within each of these columns, and all these nodes will be merged into a single node. Other incoming edges that go into the starting node and other outgoing edges that go out of the final node are allowed.

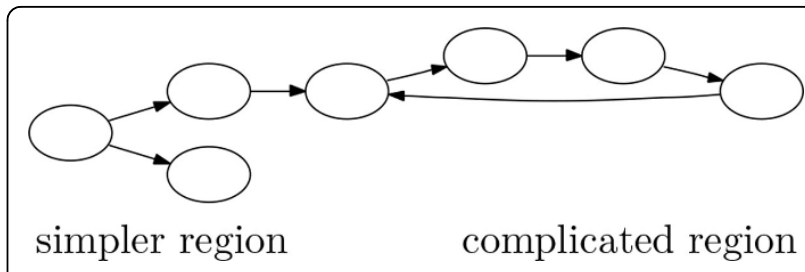

Figure 3 Example of the decomposition of a connected component. Each of the three edges on the left-hand side is a strongly connected component by itself, and the subgraph containing these three edges represents the simpler region. The cycle on the right-hand side is one single strongly connected component that represents the complicated region.

step can be performed by depth-first search with running time that is proportional to the size of the graph [15].

\section{Forward-backward tangles}

Since Velvet assembles the forward and the backward strands simultaneously, each gene should be represented by two disjoint components, one on each strand, which do not contain any cycles. Although there are no more cycles after removing the strongly connected components that are not just a single edge, it is still possible to have forward-backward tangles in which a forward node and the corresponding backward node reside within the same connected component. These forward-backward tangles can be identified by depth-first search [15].

\section{Splicing graphs}

We extract all the nodes within the strongly connected components that are not just a single edge and within the forward-backward tangles. We treat each node as an individual assembly that consists only of a single node while ignoring the junction information within these complicated regions. We then remove these nodes and their adjacent edges, and extract the connected components in the remaining graph. Each of these connected components does not contain cycles and should mostly represent alternatively spliced variants of one gene. Only one of the two possible orientations is retained for each extracted node and each connected component.

\section{Junction adjustment}

Since adjacent vertices in a de Bruijn graph share overlapping sequence fragments of length $k-1$ according to the definition of a de Bruijn graph, the location of junctions is imprecise and this representation is hard to interpret. Although the overlaps can be eliminated by following the strategy in [5] to remove the first $k-1$ letters of the sequence in each node, the location of junctions is still imprecise and the beginning part of some of the sequences is missing. To resolve these 
uncertainties, we start with the non-overlapping strategy employed in [5] and consider two cases: at a split junction in which a path branches in more than one direction, the junction is precise and no change is necessary; at a merge junction in which more than one path meets at a node, we move the maximum number of shared letters in the suffix part of these paths to the meeting node to make the junction precise (see Figure 4 for an example).

Note that there can still be ambiguities due to the presence of repeating patterns across junctions. Since the graph no longer contains cycles after the previous processing steps, we recover the first $k-1$ letters in each starting node with no incoming edges by restoring the removed letters. After these adjustments, we consider each resulting component as a splicing graph that specifies the alternative splicing paths of one gene. Note that we only resolve simple cases and do nothing when there are simultaneously a split and a merge at the two ends of an edge. To remove very short assemblies, we retain only the splicing graphs in which all paths from a source to a sink have sequence length above $2 k-1$.

\section{De novo expression estimate}

In order to evaluate differential expression levels in a non-model organism in which no prior information is available, we employ a measure of number of reads per kilobase of node per million reads (RPKM) [11] that is similar to the statistics used by [16] and [17]. Since there is no information about exons in a de novo assembly, reads that appear in the assembly are used instead of mapped reads. Also, each node in a splicing graph is evaluated instead of each exon, with each read that contains a $k$-mer within a node contributing to that node. Within each node, a RPKM estimate is reported independently for each library within the same assembly. A validation of the de novo RPKM values was given in [11] that shows strong correlations of these values with the ones given by Cufflinks [18] on genes without alternative splicing and good correlations on nodes from genes with alternatively splicing.

\section{Postprocessing software}

A software program implementing our postprocessing algorithm is available at http://faculty.cse.tamu.edu/ shsze/postprocess. In order to make the results directly applicable to other software during downstream analysis, we represent each assembly in an annotated FASTA format, in which each potentially non-linear structure is represented by a collection of nodes, with connecting edge information and RPKM values for each library embedded within the name of each node.

\section{Results and discussion}

\section{Drosophila melanogaster simulations}

To simulate the transcriptome assembly of Drosophila, we extracted all gene transcripts from the $D$. melanogaster genome. For each gene transcript, we randomly pick reads until an average nucleotide coverage of 100 is reached while allowing varying percentages of mismatches in the reads, giving 70598749 reads of length 75 .

We applied Velvet by setting the parameters max_branch_length, max_divergence and max_gap_count to 0 , while enabling read_trkg. We performed assemblies over different values of hash length $k$ and cov_cutoff $c$. We extracted the de Bruijn graph from the LastGraph file and applied our postprocessing algorithm. Since de novo sequence assembly is performed mostly on nonmodel organisms and possible function of the assembled sequences is accessed with respect to a closely related organism, we used translated BLAST search [19] to simulate its usage.

While it is possible to recover $90 \%$ of the Drosophila genes under ideal conditions when there are no mismatches in the reads [11], Table 1 shows that the assemblies were still of high quality for $0.1 \%$ mismatches, with more than $73 \%$ of genes recovered. Table 2 shows that the performance for $0.2 \%$ mismatches was much worse, with only about half of the genes recovered. When $k$ is small, the larger number of nodes in the simulated Drosophila assemblies can contain more information, although a larger proportion of them were in tangles and they were more likely to be in complicated regions. The

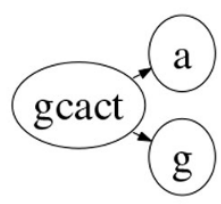

split: no change

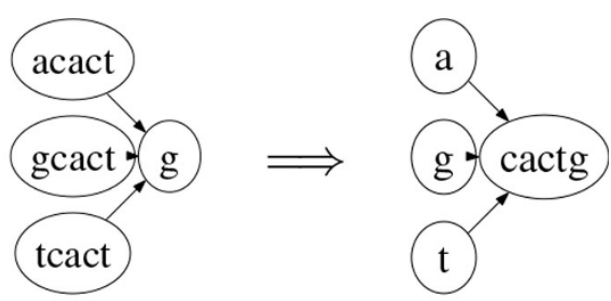

merge: adjusted

Figure 4 Example of junction adjustment. 
Table 1 Statistics of the simulated transcriptome assemblies of Drosophila using its known complete genome over different values of $k$ and $k$-mer coverage cutoff $c$ with $0.1 \%$ mismatches in the reads.

\begin{tabular}{|c|c|c|c|c|c|c|c|c|c|c|c|c|c|c|c|c|}
\hline$k \_c$ & $\begin{array}{l}\text { initial } \\
\text { nodes }\end{array}$ & $\begin{array}{c}\text { largest } \\
\text { tangle }\end{array}$ & $\begin{array}{l}\text { largest } \\
\text { SCC }\end{array}$ & $\begin{array}{c}\text { splicing } \\
\text { graphs }\end{array}$ & $\begin{array}{c}\text { max } \\
\text { length }\end{array}$ & N50 & $\begin{array}{c}>1- \\
\text { node } \\
\text { graphs }\end{array}$ & $\begin{array}{c}\max \\
\text { nodes }\end{array}$ & $\begin{array}{c}\text { avg } \\
\text { nodes }\end{array}$ & SNPs & $\begin{array}{l}\text { total } \\
\text { hits }\end{array}$ & $\begin{array}{l}\text { unique } \\
\text { hits }\end{array}$ & $\begin{array}{l}>1 \text {-hit } \\
\text { graphs }\end{array}$ & $\begin{array}{l}\max \\
\text { hits }\end{array}$ & $\begin{array}{c}\text { time } \\
\text { (mins) }\end{array}$ & $\begin{array}{c}\text { memory } \\
\text { (GB) }\end{array}$ \\
\hline 25_3 & 38884 & 17900 & 9937 & 15713 & 37380 & 2366 & 1361 & 3106 & 10 & 883 & 12731 & 10162 & 643 & 27 & 80,3 & 21,2 \\
\hline 25_5 & 34822 & 16979 & 9255 & 15521 & 37380 & 2374 & 1351 & 266 & 7 & 517 & 12708 & 10160 & 643 & 27 & 80,3 & 21,2 \\
\hline 25_10 & 34494 & 16712 & 9057 & 15486 & 37380 & 2373 & 1345 & 194 & 7 & 481 & 12699 & 10158 & 639 & 27 & 80,3 & 21,2 \\
\hline 31_3 & 28342 & 5037 & 2080 & 13819 & 45158 & 2704 & 1719 & 1007 & 7 & 496 & 12523 & 11112 & 546 & 12 & 76,3 & 18,2 \\
\hline 31_5 & 27307 & 4971 & 1898 & 13740 & 45158 & 2714 & 1717 & 167 & 6 & 381 & 12494 & 11110 & 552 & 13 & 76,3 & 18,2 \\
\hline 31_10 & 27265 & 4947 & 1885 & 13829 & 45158 & 2704 & 1698 & 161 & 6 & 377 & 12536 & 11109 & 542 & 13 & 76,3 & 18,2 \\
\hline
\end{tabular}

Initial nodes denotes the number of nodes that are in the initial assembly. Largest tangle denotes the number of nodes of the largest connected component. Largest SCC denotes the number of nodes of the largest strongly connected component. Splicing graphs denotes the number of splicing graphs. Max length denotes the length (in nucleotides) of the longest path over all splicing graphs. N50 denotes the N50 value of the length (in nucleotides) of the longest path in each graph. >1-node graphs denotes the number of graphs with more than one node. Max nodes denotes the maximum number of nodes in these non-linear graphs. Avg nodes denotes the average number of nodes in these non-linear graphs. SNPs denotes the number of SNPs recovered. Total hits denotes the total number of hits from translated BLAST search of each node to Drosophila (isoforms are considered the same gene, only the top hit with $E$-value below $10^{-7}$ is included for each node in a splicing graph, and hits from nodes within the same splicing graph to the same gene are counted once). Unique hits denotes the number of unique hits to different genes. >1-hit graphs denotes the number of splicing graphs that have BLAST hits to more than one gene. Max hits denotes the maximum number of different genes that have BLAST hits to a splicing graph. Time (mins) denotes the computational time in minutes, with the values to the left and to the right of "," indicating the running time of Velvet and our postprocessing algorithm respectively. Memory (GB) denotes the memory requirement in gigabytes, with the values to the left and to the right of "," indicating the memory requirement of Velvet and our postprocessing algorithm respectively.

Table 2 Statistics of the simulated transcriptome assemblies of Drosophila using its known complete genome over different values of $k$ and $k$-mer coverage cutoff $c$ with $0.2 \%$ mismatches in the reads. The notations are the same as in Table 1.

\begin{tabular}{|c|c|c|c|c|c|c|c|c|c|c|c|c|c|c|c|c|}
\hline$k \_c$ & $\begin{array}{l}\text { initial } \\
\text { nodes }\end{array}$ & $\begin{array}{c}\text { largest } \\
\text { tangle }\end{array}$ & $\begin{array}{l}\text { largest } \\
\text { SCC }\end{array}$ & $\begin{array}{l}\text { splicing } \\
\text { graphs }\end{array}$ & $\begin{array}{c}\max \\
\text { length }\end{array}$ & N50 & $\begin{array}{c}>1- \\
\text { node } \\
\text { graphs }\end{array}$ & $\begin{array}{c}\max \\
\text { nodes }\end{array}$ & $\begin{array}{c}\text { avg } \\
\text { nodes }\end{array}$ & SNPs & $\begin{array}{l}\text { total } \\
\text { hits }\end{array}$ & $\begin{array}{l}\text { unique } \\
\text { hits }\end{array}$ & $\begin{array}{l}>1 \text {-hit } \\
\text { graphs }\end{array}$ & $\begin{array}{l}\max \\
\text { hits }\end{array}$ & $\begin{array}{c}\text { time } \\
\text { (mins) }\end{array}$ & $\begin{array}{l}\text { memory } \\
\text { (GB) }\end{array}$ \\
\hline 25_3 & 45305 & 23504 & 15883 & 13240 & 26909 & 2255 & 634 & 8671 & 27 & 2049 & 8258 & 6188 & 315 & 16 & 94,3 & 30,2 \\
\hline 25_5 & 29090 & 16349 & 11411 & 11734 & 27251 & 2321 & 606 & 1832 & 11 & 337 & 8156 & 6180 & 321 & 12 & 94,3 & 30,2 \\
\hline 25_10 & 26297 & 15235 & 10367 & 11606 & 27251 & 2329 & 595 & 165 & 8 & 257 & 8116 & 6176 & 319 & 13 & 94,3 & 30,2 \\
\hline $31 \_3$ & 23544 & 5604 & 2331 & 11993 & 44990 & 2536 & 583 & 1520 & 12 & 611 & 9561 & 8488 & 281 & 17 & 83,3 & 21,2 \\
\hline $31 \_5$ & 19869 & 4299 & 2097 & 11650 & 44990 & 2545 & 571 & 253 & 7 & 248 & 9548 & 8488 & 281 & 13 & 83,3 & 21,2 \\
\hline 31_10 & 19541 & 4222 & 2056 & 11642 & 44990 & 2545 & 572 & 96 & 7 & 233 & 9544 & 8484 & 281 & 13 & 83,3 & 21,2 \\
\hline
\end{tabular}

extraction of strongly connected components reduced the size of the most complicated region by about half.

When $k$ is small, the larger number of splicing graphs resulted in more complete assemblies, although the sequences were shorter and thus more fragmented. When $k$ is large, the maximum and median (N50) lengths of splicing graphs approached the maximum and median lengths of gene transcripts in the known Drosophila genome, which are 69439 and 3231 respectively. Between 5 to $12 \%$ of splicing graphs had non-linear structures. These values are a significant portion of the percentage of known Drosophila genes that have more than one alternatively spliced variant, which is $27 \%$. A small number of SNPs were recovered, which may be due to variations in repeats or the inability to separate gene families.

When compared to the total number of BLAST hits, the number of unique BLAST hits to different Drosophila genes was not much smaller. When compared to the total number of splicing graphs, only a small number of graphs have BLAST hits to more than one gene. Within these graphs, the maximum number of different genes that have BLAST hits to a graph was small, thus we have mostly achieved the goal that each splicing graph should represent alternatively spliced variants of only one gene. When the $k$-mer coverage cutoff $c$ is 3 , the number of junctions and some of the splicing graphs were very large. Otherwise, the results were similar over different cutoffs for the same value of $k$. This is due to the consistent high coverage that is guaranteed by the simulation.

Figure 5 and Figure 6 show that while the sensitivity with respect to protein sequence BLAST and alternative splicing junctions decreases as the percentage of mismatches increases, the specificity remained high. For $0.2 \%$ mismatches, only about half of the coding positions and about $20 \%$ of the alternative splicing junctions were recovered, indicating that it is much harder to recover the alternative splicing junctions. 

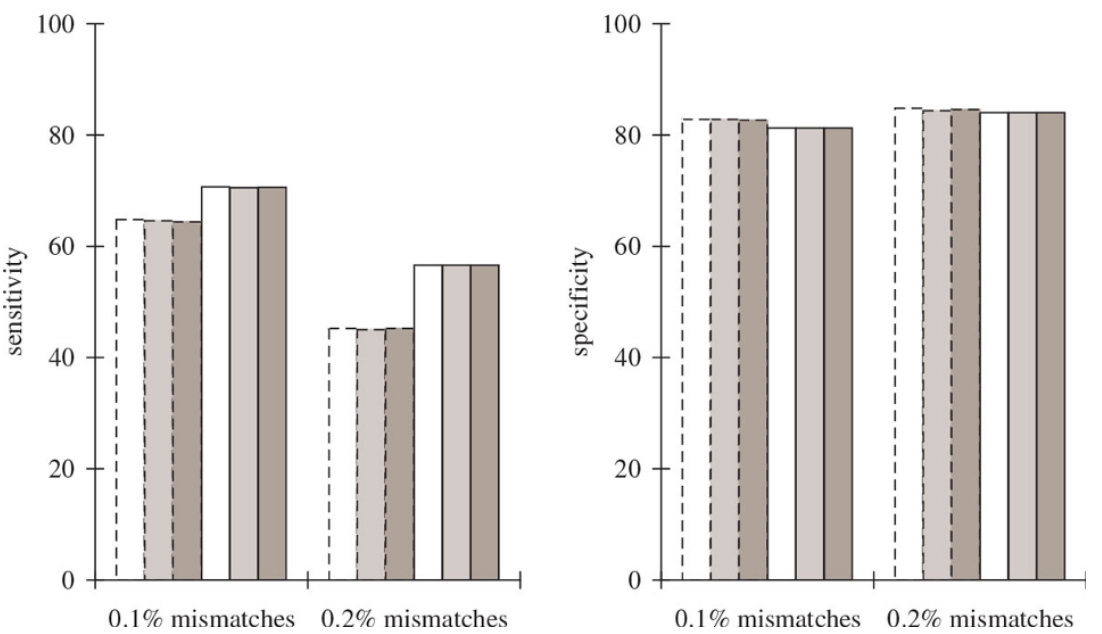

Figure 5 Comparisons of the protein sequence BLAST results in the simulated transcriptome assemblies of Drosophila using its known complete genome over different values of $k$ and $k$-mer coverage cutoff $c$ (represented by $k \_c$ ) with varying percentages of mismatches in the reads. Sensitivity is defined to be the percentage of coding positions in the genome that are recovered in the assembly considering only Drosophila gene transcripts that are found in BLAST hits (each position that is within some coding region is counted once). Specificity is defined to be the percentage of predicted transcript positions in the assembly that are included in BLAST alignments considering only predictions that have BLAST hits.

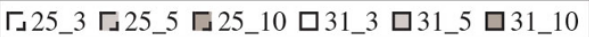
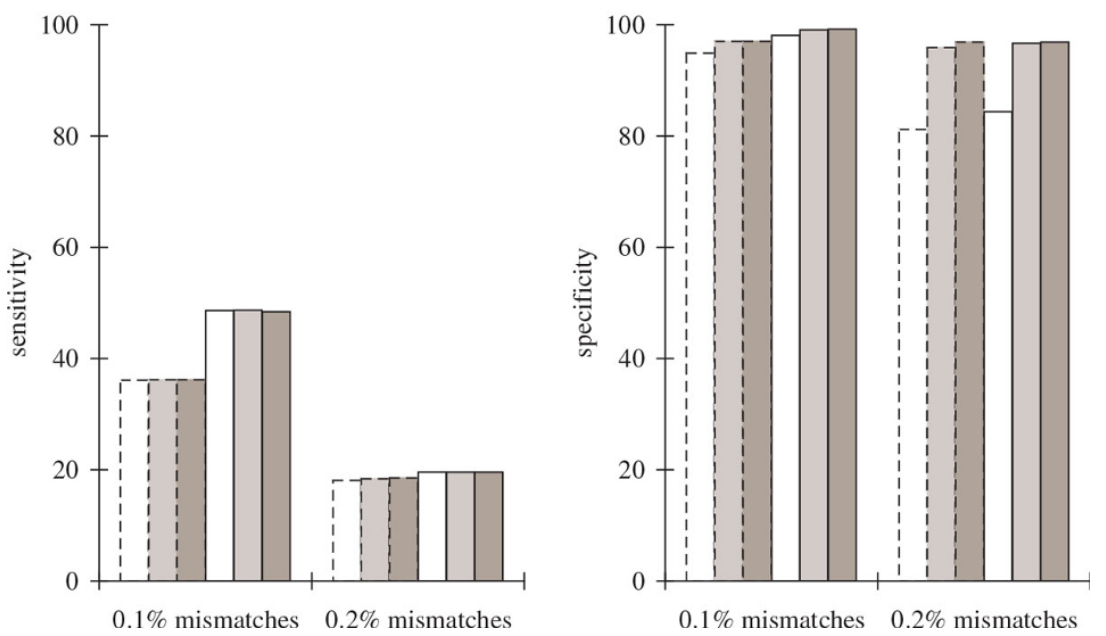

Figure 6 Comparisons of the alternative splicing junction results in the simulated transcriptome assemblies of Drosophila using its known complete genome over different values of $k$ and $k$-mer coverage cutoff $c$ (represented by $k_{-} c$ ) with varying percentages of mismatches in the reads. Sensitivity is defined to be the percentage of junctions in the gene transcripts of Drosophila that appear somewhere in the assembly. Specificity is defined to be the percentage of junctions in the assembly that appear somewhere in the gene transcripts of Drosophila. Junctions in the gene transcripts of Drosophila are defined by concatenating the two sequences of length $k$ that are immediately to the left and immediately to the right of all alternative splicing locations to obtain a sequence of length $2 k$. Junctions in the assembly are defined by concatenating the two $k$-mers at the beginning and ending nodes of an edge to obtain a sequence of length $2 k$ after the elimination of overlapping sequence fragments between adjacent nodes. Up to three mismatches are allowed when looking for these sequence occurrences. 


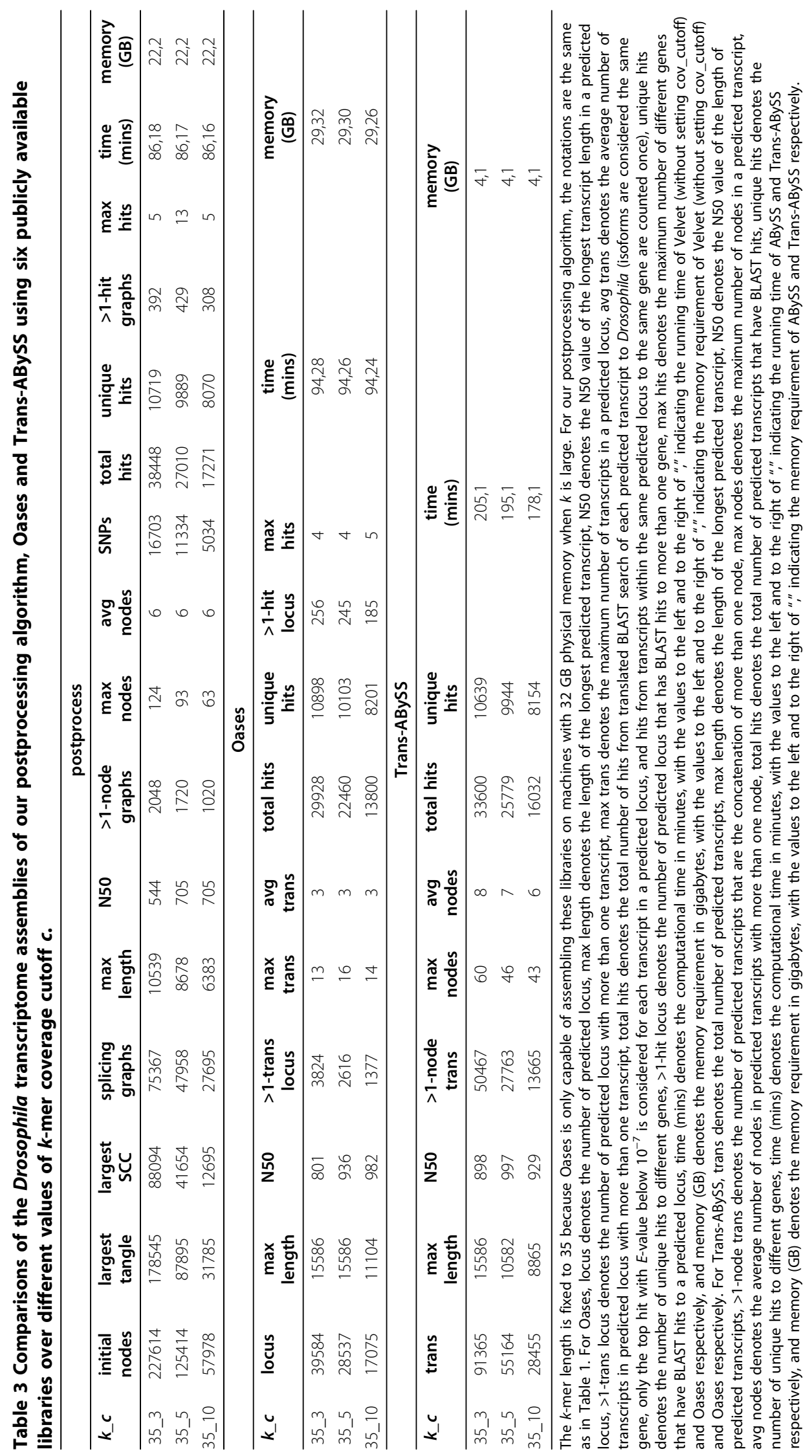




\section{Drosophila public libraries}

To investigate the transcriptome assembly of Drosophila under realistic conditions, we obtained reads of length 75 from six RNA-Seq libraries in [20] at the sequence read archive [21] that include the following developmental stages: 2-16 hours embryos (SRX019647), third instar larvae (SRX019648), mixed pupae (SRX019651, two replicates), adult females (SRX019652), and adult males (SRX019653). Since sequence quality decreases toward the end of a read, we trimmed each read by removing all positions including and to the right of the first position that has a quality score of less than 15 , giving a

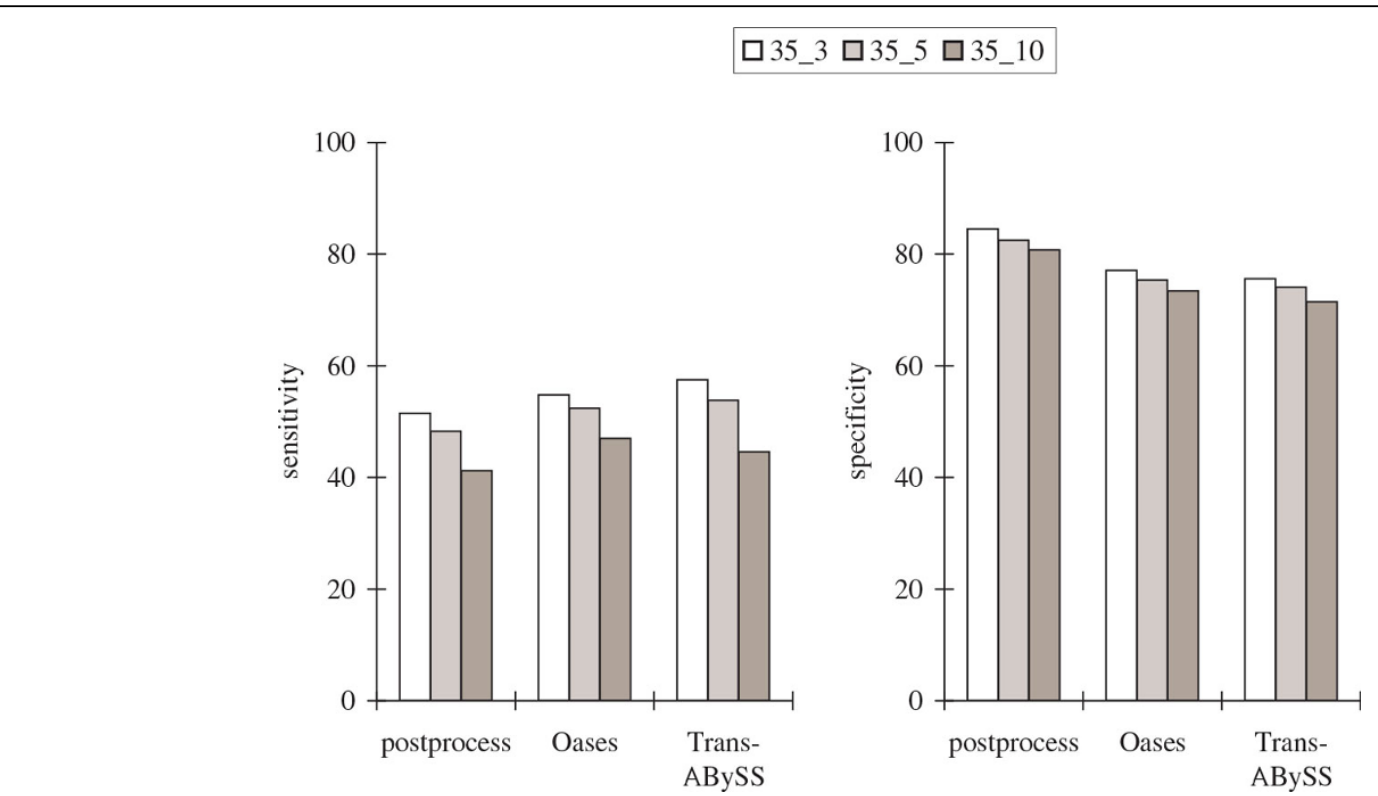

Figure 7 Comparisons of the protein sequence BLAST results in the Drosophila transcriptome assemblies using six publicly available libraries with $\boldsymbol{k}=\mathbf{3 5}$ and over different values of $\boldsymbol{k}$-mer coverage cutoff $\boldsymbol{c}$ (represented by $\boldsymbol{k}_{-} \boldsymbol{c}$ ). The notations are the same as in Figure 5 .

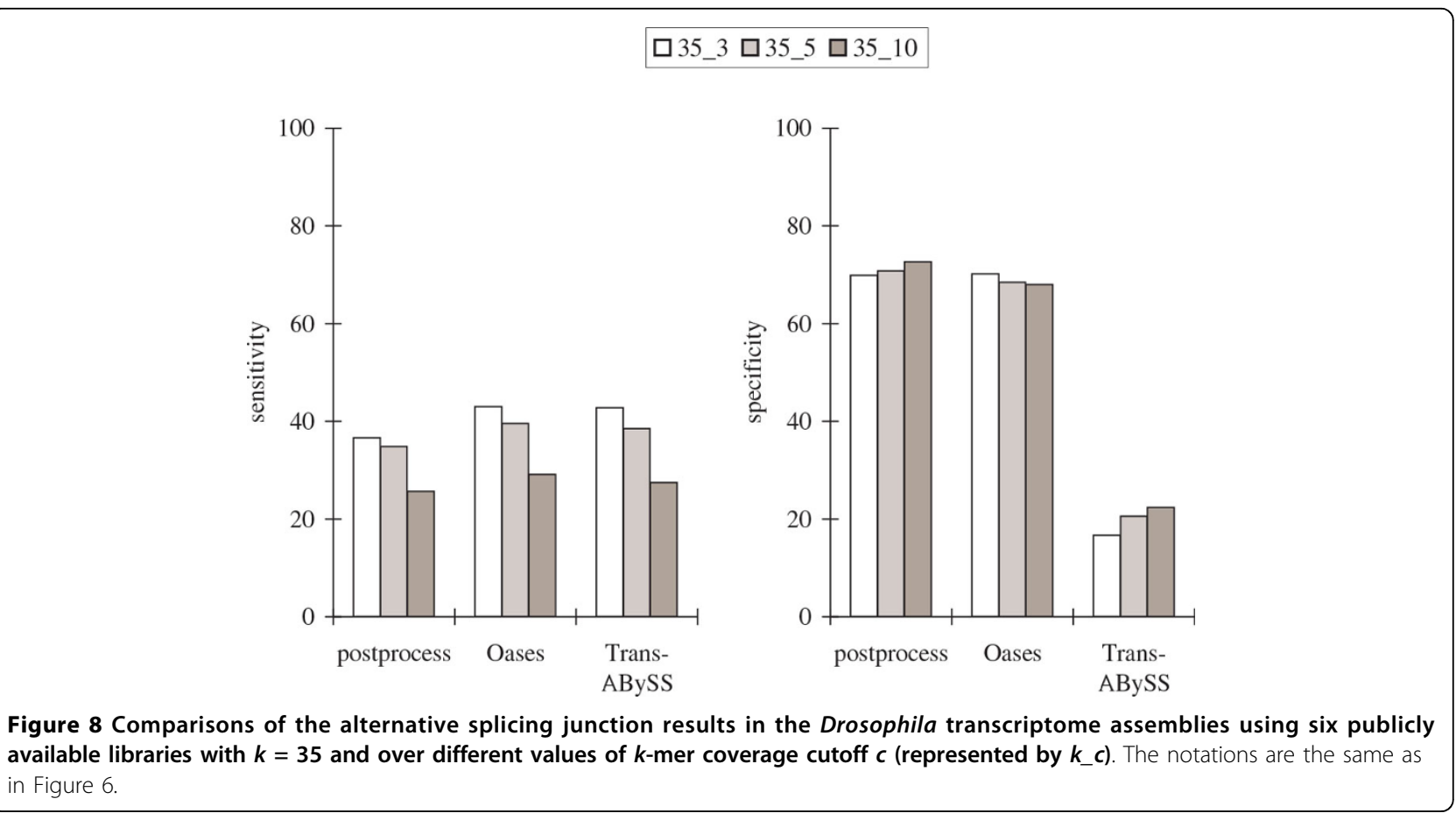




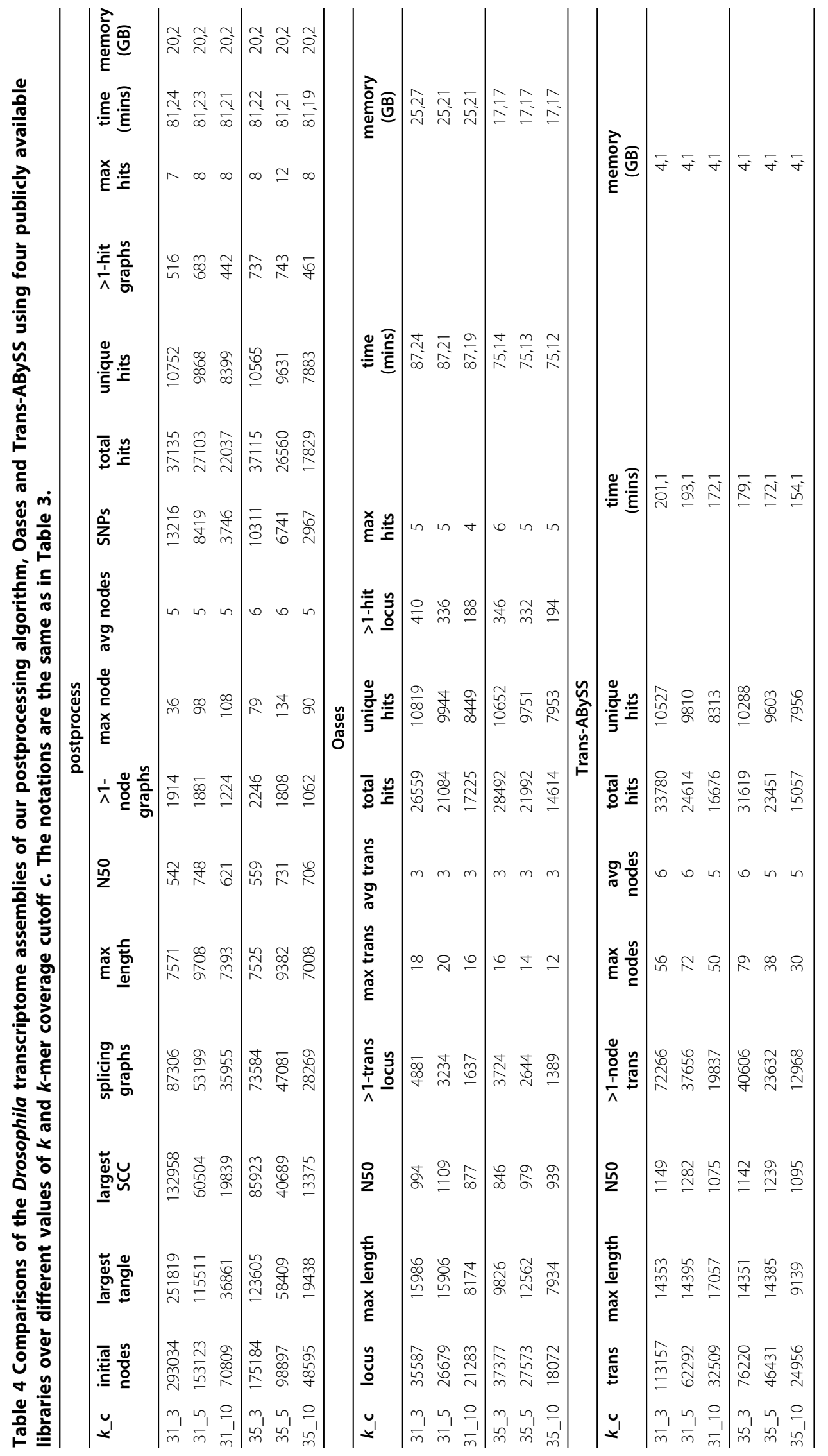


total of 102262392 reads with average length 40 . We compare the performance of our postprocessing algorithm to Oases and Trans-ABySS on machines with 32 GB physical memory. Since the memory requirement of Oases exceeds 32 GB when the $k$-mer length is small, we fix $k$ to 35 .

For Oases, Velvet was applied with hash length $k$ without setting cov_cutoff while enabling read_trkg. Oases was then applied on the results from Velvet with cov_cutoff $c$. For Trans-ABySS, abyss-pe was applied with $k$-mer size $k$, mean $k$-mer coverage threshold $\mathrm{c}$, and minimum number of pairs $n=10$. Trans-ABySS was then applied on the results from abyss-pe by utilizing the assembly.py script with the single $k$-mer length. For our postprocessing algorithm and Oases, all reads were treated as single-end reads.

Table 3 shows that the maximum and median lengths of the assemblies were much smaller for all the three algorithms when compared to the simulation results. The number of unique BLAST hits decreases as the $k$ mer coverage cutoff $c$ increases, while the ratio of the total number of BLAST hits to the number of number of unique BLAST hits was between 1.5 to 4, indicating sequence fragmentation. Trans-ABySS had the longest assemblies, while Oases had the largest number of unique BLAST hits. Although our postprocessing algorithm had the shortest assemblies, it had a larger number of unique BLAST hits than Trans-ABySS when the $k$-mer coverage cutoff $c$ is 3 .

Figure 7 and Figure 8 show that although our postprocessing algorithm had the lowest sensitivity with respect to protein sequence BLAST and alternative splicing junctions, it had the highest sequence specificity. Both our postprocessing algorithm and Oases had the highest specificity with respect to alternative splicing junctions, although it was not as high when compared to the simulation results.

In order to evaluate the performance with varying $k$, we also considered a smaller set of four libraries by removing the second replicate from the mixed pupal libraries and replacing the two adult libraries with a single adult library at a different time point (SRX019657), giving a total of 76819166 reads with average length 42 after quality trimming. Table 4, Figure 9 and Figure 10 show that the results had similar characteristics as the ones with six libraries, with better performance when $k=31$.

\section{Conclusions}

We have developed a postprocessing algorithm that can recover alternative splicing information directly from de Bruijn graphs of RNA-Seq data. Our strategy does not require prior genomic knowledge and supports the study of differential expression through investigating de novo RPKM values [11]. The computational time is linear in the size of the de Bruijn graph, and our algorithm takes a few minutes to half-an-hour to complete after results from Velvet are available in the test cases (see Tables 1-4). It uses less memory than Velvet, while running Oases together with Velvet without setting cov_cutoff is often more memory-intensive than running Velvet with cov_cutoff (see Tables 1-4). Our algorithm performs well on simulations with low percentages of mismatches in the reads and generally has higher specificity than Oases or Trans-ABySS. It is most suitable in situations in which a more reliable assembly is desired at the expense of lower sensitivity. Our algorithm has been applied to perform transcriptome assembly of the non-model blow fly Lucilia sericata in [11], which allows comparison of its transcriptome to the closely related model organism Drosophila through translated

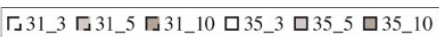
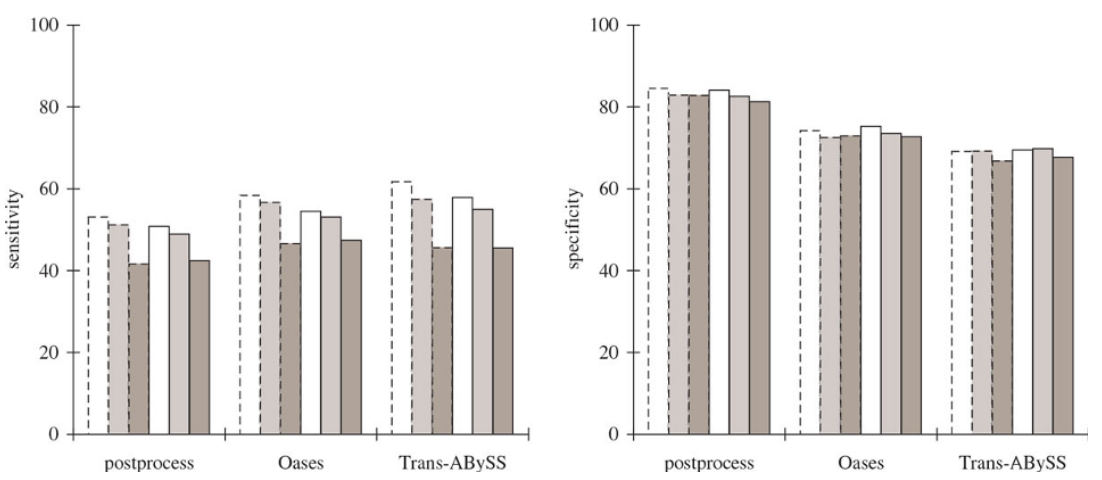

Figure 9 Comparisons of the protein sequence BLAST results in the Drosophila transcriptome assemblies using four publicly available libraries over different values of $\boldsymbol{k}$ and $\boldsymbol{k}$-mer coverage cutoff $\boldsymbol{c}$ (represented by $\boldsymbol{k} \_\boldsymbol{c}$ ). The notations are the same as in Figure 5 . 

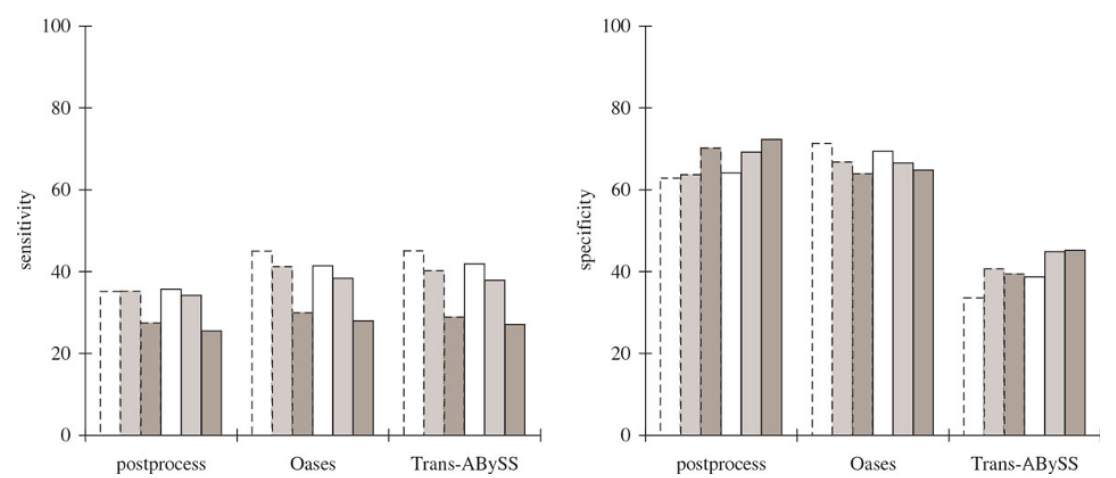

Figure 10 Comparisons of the alternative splicing junction results in the Drosophila transcriptome assemblies using four publicly available libraries over different values of $\boldsymbol{k}$ and $\boldsymbol{k}$-mer coverage cutoff $\boldsymbol{c}$ (represented by $\boldsymbol{k} \_\boldsymbol{c}$ ). The notations are the same as in Figure 6 .

BLAST search, investigation of alternative splicing and differential expression among various developmental stages, and identification of transposable elements.

\section{Competing interests}

The authors declare that they have no competing interests.

\section{Authors' contributions}

AMT and S-HS designed the computational work, analyzed the data and wrote the paper. S-HS did the simulation experiments. All authors read and approved the final manuscript.

\section{Acknowledgements}

Computations were performed on the Brazos Cluster at Texas A\&M University. AMT was supported by startup funds from the College of Agriculture and Life Sciences at Texas A\&M University and Texas Agrilife Research. S-HS was supported by NSF grant MCB-0951120. This work was supported in part by NIJ grant 2012-DN-BX-K024. Points of view in this document are those of the authors and do not necessarily represent the official position or policies of the U.S. Department of Justice.

\section{Declarations}

Publication costs for this work were funded by the Open Access to Knowledge (OAK) Fund at the Texas A\&M University Libraries. This article has been published as part of BMC Genomics Volume 15 Supplement 5, 2014: Selected articles from the Third IEEE International Conference on Computational Advances in Bio and Medical Sciences (ICCABS 2013): Genomics. The full contents of the supplement are available online at http://www.biomedcentral.com/bmcgenomics/ supplements/15/S5

\section{Authors' details}

'Department of Computer Science and Engineering, Texas A\&M University, College Station, TX 77843, USA. ${ }^{2}$ Department of Biochemistry \& Biophysics, Texas A\&M University, College Station, TX 77843, USA. ${ }^{3}$ Department of Entomology, Texas A\&M University, College Station, TX 77843, USA.

Published: 14 July 2014

\section{References}

1. Dohm JC, Lottaz C, Borodina T, Himmelbauer H: SHARCGS, a fast and highly accurate short-read assembly algorithm for de novo genomic sequencing. Genome Res 2007, 17:1697-1706.

2. Butler J, MacCallum I, Kleber M, Shlyakhter IA, Belmonte MK, Lander ES, Nusbaum C, Jaffe DB: ALLPATHS: de novo assembly of whole-genome shotgun microreads. Genome Res 2008, 18:810-820.
3. Chaisson MJ, Pevzner PA: Short read fragment assembly of bacterial genomes. Genome Res 2008, 18:324-330.

4. Hernandez D, François $P$, Farinelli L, Østerås $M$, Schrenzel J: De novo bacterial genome sequencing: millions of very short reads assembled on a desktop computer. Genome Res 2008, 18:802-809.

5. Zerbino DR, Birney E: Velvet: algorithms for de novo short read assembly using de Bruijn graphs. Genome Res 2008, 18:821-829.

6. Birol I, Jackman SD, Nielsen CB, Qian JQ, Varhol R, Stazyk G, Morin RD, Zhao Y, Hirst M, Schein JE, Horsman DE, Connors JM, Gascoyne RD, Marra MA, Jones SJM: De novo transcriptome assembly with ABySS. Bioinformatics 2009, 25:2872-2877.

7. Li R, Zhu H, Ruan J, Qian W, Fang X, Shi Z, Li Y, Li S, Shan G, Kristiansen K, Li S, Yang H, Wang J, Wang J: De novo assembly of human genomes with massively parallel short read sequencing. Genome Res 2010, 20:265-272.

8. Grabherr MG, Haas BJ, Yassour M, Levin JZ, Thompson DA, Amit I, Adiconis X, Fan L, Raychowdhury R, Zeng Q, Chen Z, Mauceli E, Hacohen N, Gnirke A, Rhind N, di Palma F, Birren BW, Nusbaum C, Lindblad-Toh K, Friedman N, Regev A: Full-length transcriptome assembly from RNA-Seq data without a reference genome. Nat Biotechnol 2011, 29:644-652.

9. Robertson G, Schein J, Chiu R, Corbett R, Field M, Jackman SD, Mungall K, Lee S, Okada HM, Qian JQ, Griffith M, Raymond A, Thiessen N, Cezard T, Butterfield YS, Newsome R, Chan SK, She R, Varhol R, Kamoh B, Prabhu AL, Tam A, Zhao Y, Moore RA, Hirst M, Marra MA, Jones SJM, Hoodless PA, Birol I: De novo assembly and analysis of RNA-seq data. Nat Methods 2010, 7:909-912.

10. Schulz MH, Zerbino DR, Vingron M, Birney E: Oases: robust de novo RNAseq assembly across the dynamic range of expression levels. Bioinformatics 2012, 28:1086-1092.

11. Sze SH, Dunham JP, Carey B, Chang PL, Li F, Edman RM, Fjeldsted C, Scott MJ, Nuzhdin SV, Tarone AM: A de novo transcriptome assembly of Lucilia sericata (Diptera: Calliphoridae) with predicted alternative splices, single nucleotide polymorphisms, and transcript expression estimates. Insect Mol Biol 2012, 21:205-221.

12. Pevzner PA: I-tuple DNA sequencing: computer analysis. J Biomol Struct Dyn 1989, 7:63-73.

13. Idury RM, Waterman MS: A new algorithm for DNA sequence assembly. J Comput Biol 1995, 2:291-306.

14. Heber S, Alekseyev M, Sze SH, Tang H, Pevzner PA: Splicing graphs and EST assembly problem. Bioinformatics 2002, 18:S181-188.

15. Cormen TH, Leiserson CE, Rivest RL, Stein C: Introduction to Algorithms, Second Edition The MIT Press; 2001.

16. Mortazavi A, Williams BA, McCue K, Schaeffer L, Wold B: Mapping and quantifying mammalian transcriptomes by RNA-Seq. Nat Methods 2008, 5:621-628.

17. Trapnell C, Pachter L, Salzberg SL: TopHat: discovering splice junctions with RNA-Seq. Bioinformatics 2009, 25:1105-1111.

18. Trapnell C, Williams BA, Pertea G, Mortazavi A, Kwan G, van Baren MJ, Salzberg SL, Wold BJ, Pachter L: Transcript assembly and quantification by 
RNA-Seq reveals unannotated transcripts and isoform switching during cell differentiation. Nat Biotechnol 2010, 28:511-515.

19. Altschul SF, Gish W, Miller W, Myers EW, Lipman DJ: Basic local alignment search tool. J Mol Biol 1990, 215:403-410.

20. Daines B, Wang H, Wang L, Li Y, Han Y, Emmert D, Gelbart W, Wang X, Li W, Gibbs R, Chen R: The Drosophila melanogaster transcriptome by paired-end RNA sequencing. Genome Res 2011, 21:315-324.

21. Sayers EW, Barrett T, Benson DA, Bolton E, Bryant SH, Canese K, Chetvernin V, Church DM, DiCuccio M, Federhen S, Feolo M, Geer LY, Helmberg W, Kapustin Y, Landsman D, Lipman DJ, Lu Z, Madden TL, Madej T, Maglott DR, Marchler-Bauer A, Miller V, Mizrachi I, Ostell J, Panchenko A, Pruitt KD, Schuler GD, Sequeira E, Sherry ST, Shumway M, Sirotkin K, Slotta D, Souvorov A, Starchenko G, Tatusova TA, Wagner L, Wang Y, Wilbur WJ, Yaschenko E, Ye J: Database resources of the National Center for Biotechnology Information. Nucleic Acids Res 2010, 38:D5-16.

doi:10.1186/1471-2164-15-S5-S6

Cite this article as: Sze and Tarone: A memory-efficient algorithm to obtain splicing graphs and de novo expression estimates from de Bruijn graphs of RNA-Seq data. BMC Genomics 2014 15(Suppl 5):S6.

\section{Submit your next manuscript to BioMed Central} and take full advantage of:

- Convenient online submission

- Thorough peer review

- No space constraints or color figure charges

- Immediate publication on acceptance

- Inclusion in PubMed, CAS, Scopus and Google Scholar

- Research which is freely available for redistribution

Submit your manuscript at www.biomedcentral.com/submit 